\title{
The Lynx X-ray Observatory: Concept Study Overview and Status
}

Jessica A. Gaskin*a, Alexandra Dominguez ${ }^{\mathrm{a}}$, Karen Gelmis ${ }^{\mathrm{a}}$, John (Jack) Mulqueen a , Douglas Swartz $^{\mathrm{b}}$, Kevin McCarley ${ }^{\mathrm{a}}$, Feryal Özel ${ }^{\mathrm{c}}$, Alexey Vikhlinin ${ }^{\mathrm{d}}$, Daniel Schwartz ${ }^{\mathrm{d}}$, Harvey Tananbaum ${ }^{\mathrm{d}}$, Gary Blackwood ${ }^{\mathrm{e}}$, Jonathan Arenberg ${ }^{\mathrm{f}}$, William Purcell ${ }^{\mathrm{g}}$, Lynn Allen ${ }^{\mathrm{h}}-$ On behalf of the Lynx Team

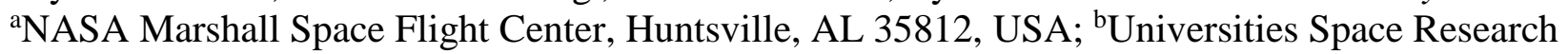
Association Science and Technology Institute, Huntsville, AL 35805, USA, 'University of Arizona, Tucson, AZ, 85721, USA, 'S mithsonian Astrophysical Observatory, Cambridge, MA 02138, USA, ${ }^{e}$ NASA Jet Propulsion Laboratory, Pasadena, CA 91109, USA, Northrop Grumman, Aerospace

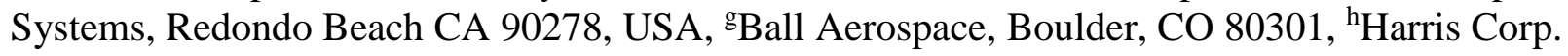
Space and Intelligence Systems, Rochester, NY 14624, USA

\begin{abstract}
Lynx, one of four strategic mission concepts under study for the 2020 Astrophysics Decadal Survey, will provide leaps in capability over previous and planned X-ray missions, and will provide synergistic observations in the 2030s to a multitude of space- and ground-based observatories across all wavelengths. Lynx will have orders of magnitude improvement in sensitivity, on-axis sub-arcsecond imaging with arcsecond angular resolution over a large field of view, and high-resolution spectroscopy for point-like and extended sources. The Lynx architecture enables a broad range of unique and compelling science, to be carried out mainly through a General Observer Program. This Program is envisioned to include detecting the very first supermassive black holes, revealing the high-energy drivers of galaxy and structure formation, characterizing the mechanisms that govern stellar activity - including effects on planet habitability, and exploring the highest redshift galaxy clusters. An overview and status of the Lynx concept are summarized.
\end{abstract}

Keywords: Lynx X-ray Observatory, X-Ray Astronomy, Strategic Mission Concept, 2020 Astrophysics Decadal

\section{INTRODUCTION}

Two years ago, four large strategic mission concepts, based on those defined in the Astrophysics Roadmap - Enduring Quests, Daring Visions ${ }^{1}$, were selected by the astronomy community to be studied for prioritization in the 2020 Astrophysics Decadal Survey. Of these missions, Lynx, formerly X-Ray Surveyor, is the only concept that would enable the next generation of high-energy observations of the Universe. Since being selected for study, the Lynx concept has evolved into a streamlined general-purpose observatory capable of performing science befitting that of a flagship mission for a cost that would allow for a balanced Astrophysics portfolio.

\subsection{Fascinating Observations}

Rooted in the X-ray band, Lynx will operate in the $0.2 \mathrm{keV}$ to $\sim 10 \mathrm{keV}$ energy range and boasts a 50 -fold increase in sensitivity compared to the currently orbiting Chandra X-Ray Observatory, and 100-fold increase in sensitivity compared to ESA's planned Athena. These gains are possible by coupling high-angular resolution with high throughput. Lynx will also have 16 times larger field of view (FOV) for sub-arcsecond imaging and 10-20 times higher spectral resolution for both point-like and extended sources. This performance is made possible by several key technologies that have significantly matured over the past decade and includes relatively thin, lightweight X-ray mirrors, large-scale active pixel sensors, X-ray microcalorimeters, and high-resolution gratings. These technologies that have a clear path for maturation within a decadal-driven timescale, lowering risk and ultimately the cost of such an ambitious mission.

The Lynx architecture is motivated by a dynamic science case to observe the first supermassive black holes and to understand the critical drivers of galaxy and stellar formation and evolution; phenomena only evident at high-energies. Lynx couples a large area, high-resolution, mirror assembly with good wide-field performance $(0.5$ arcsecond Half Power Diameter (HPD) on-axis, < 1 arcsecond to $\sim 10$ arcminutes) to a suite of three science instruments that includes 
the High Definition X-ray Imager (HDXI) $)^{2,3,4}$, the Lynx X-ray Microcalorimeter (LXM) ${ }^{5}$, and the X-ray Grating Spectrometer (XGS $)^{6,7}$. Multiple candidate technologies for the Lynx mirrors exist, three of which are being studied in detail by the Lynx Team. These optics technologies are Full-Shell Optics developed by Brera and Marshall Spaceflight Center (MSFC) ${ }^{8,9}$, Silicon Meta-shell Optics developed by Goddard Spaceflight Center (GSFC) ${ }^{10}$, and Adjustable Segmented Optics developed by the Smithsonian Astrophysical Observatory (SAO) ${ }^{11}$.

\subsection{Designing an Observatory}

The spacecraft and mission design leverage existing heritage and benefit from simplicity of operation. Chandra, for example, has produced many Lessons Learned ${ }^{12}$ that Lynx will incorporate. The spacecraft design and mission operations borrow heavily from the Chandra Observatory, the only X-ray observatory to date to achieve sub-arcsecond angular resolution ${ }^{13}$. Lynx maneuvers and operational procedures on-orbit are close to identical to Chandra's, and similar design approaches target longevity. Chandra has been operating for nearly 20 years, and has maintained a robust science program throughout ${ }^{14}$. Lynx will have a baseline mission lifetime of 5 years, extendable to 20 years without servicing (though accommodation will be made to facilitate servicing as mandated by Congress ${ }^{15}$ ).

Lynx is a much more ambitious observatory (e.g., larger effective area and more advanced science instruments) than Chandra, and will operate in a different environment (halo orbit around Sun-Earth L2 versus highly elliptical orbit around the Earth). As such, Lynx will take advantage of many recent advances in the current state-of-the-art in the focal plane design, propulsion systems, power system, avionics, and command and data handling. A Cooperative agreement Notice (CAN) was awarded to a team at Northrup Grumman Aerospace Systems (NGAS), Ball Aerospace, and Harris Corporation to assess the design approach as it relates to the use of heritage ${ }^{16}$.

\subsection{State of Readiness}

A preliminary Program schedule for the Lynx has Phase A starting in 2024, leading to a launch in the mid- 2030s. Each of the Lynx technologies has a clear development and maturation path for reaching a Technology Readiness Level of 6 by the Project Preliminary Design Review in 2028 and for meeting Critical Design Review in 2030. All of the Lynx enabling technologies are currently at a TRL of 3 or higher, and it is expected that all will be at or approaching a TRL 4 by mid- 2020. Each of these technologies is being funded through NASA competed opportunities or directed funding, internal institutional funding, and other pre-flight Programs.

Manufacturing the hundreds to thousands of Lynx X-ray Mirrors is on the critical path and steps must be taken to balance the cost, schedule and risk for this effort. A CAN was awarded to NGAS and Harris to perform a cost, schedule, and risk assessment of the manufacturing aspects of the various mirror designs considered by the Lynx concept study ${ }^{17}$.

\section{LYNX X-RAY TELESCOPE}

The Lynx telescope design flows directly from the science requirements established by the Science and Technology Definition Team supported by a large number of Science Working Group members. Since reporting on the status of the Lynx concept in $2017^{18}$, the Lynx pillars, which are used to define the observatory architecture and define the mission requirements, now includes a broad topic regarding stellar evolution and ecosystems. The three science pillars, to be primarily carried out under a General Observer (GO) program, include:

The Dawn of Black Holes: Massive black holes start to form as early as their host galaxies. Lynx will find the first supermassive black holes in the first galaxies detected by JWST, trace their growth from the seed phase, and shed light on how they subsequently co-evolve with the host galaxies. Reaching into the seed regime in the early Universe requires $\mathrm{X}$-ray sensitivities of $\sim 10^{-19} \mathrm{erg} \mathrm{s}^{-1} \mathrm{~cm}^{-2}$. These observations require Lynx to have a large effective area (around $2 \mathrm{~m}^{2}$ at $1 \mathrm{keV}$ ) and large FOV with sub-arcsecond or better angular resolution.

The Invisible Drivers of Galaxy Formation and Evolution: The assembly, growth, and state of visible matter in cosmic structures are largely driven by violent processes that produce and disperse large amounts of energy and metals into the surrounding medium. In galaxies at least as massive as the Milky Way, the relevant baryonic component is heated and ionized to X-ray temperatures. Only Lynx will be capable of mapping this hot gas around galaxies and in the Cosmic Web, as well as characterizing in detail all significant modes of energy feedback. Essential observations require high-resolution spectroscopy ( $R \sim 5,000$ ) of background active galactic nuclei (AGNs), the ability to detect low surface brightness continuum emission, and $R \sim 2,000$ spectroscopy of extended sources on arcsecond scales - unique to Lynx. 
The Energetic Side of Stellar Evolution and Stellar Ecosystems: Lynx will probe, with unprecedented depth, a wide range of high-energy processes that provide a unique perspective on stellar birth and death, internal stellar structure, starplanet interactions, the origin of elements, and violent cosmic events. Lynx will detect X-ray emission as markers of young stars in active star forming regions, study stellar coronae in detail, and provide essential insight into the impact of stellar X-ray and extreme ultraviolet flux and winds on the habitability of their planets. Images and spectra of supernova remnants in Local Group galaxies will extend studies of stellar explosions and their aftermath to different metallicity environments. Lynx will expand our knowledge of collapsed stars through sensitive studies of X-ray binaries in galaxies as distant as $10 \mathrm{Mpc}$ and through detailed follow-ups of gravitational wave events. Lynx will greatly extend our X-ray grasp throughout the Milky Way and nearby galaxies by combining, for the first time, the required sensitivity, spectral resolution, and sharp vision to see in crowded fields.

The overall Lynx telescope design has not radically changed since previous reporting ${ }^{18}$; however, significantly higher fidelity has been designed into the observatory (Figure 1) and science instruments. The increased science instrument definition is a direct result of multiple instrument design studies involving the Lynx Instrument Working Group and the instrument design labs at both NASA MSFC and GSFC. The Lynx Mirror Assembly (LMA) includes the X-ray Mirror Assembly (XMA), retractable grating arrays, and the aft contamination cover. The contamination cover is used mainly on the ground to minimize contamination on the mirrors and gratings. Two of the science instruments, HDXI and the LXM, along with their electronics and radiators are mounted on a translation table that is part of Integrated Science Instrument Module (ISIM). Either instrument can be placed on-axis depending on the scientific objectives of a particular observation. A focus mechanism on the translation table allows for fine focus adjustment. The XGS focal plane detector assembly is mounted in a fixed location on the ISIM offset from the optical axis to intercept the dispersed spectrum regardless of whether the HDXI or LXM is at the primary focus. The XGS focal plane assembly utilizes a separate focus mechanism that is integrated into its detector assembly.

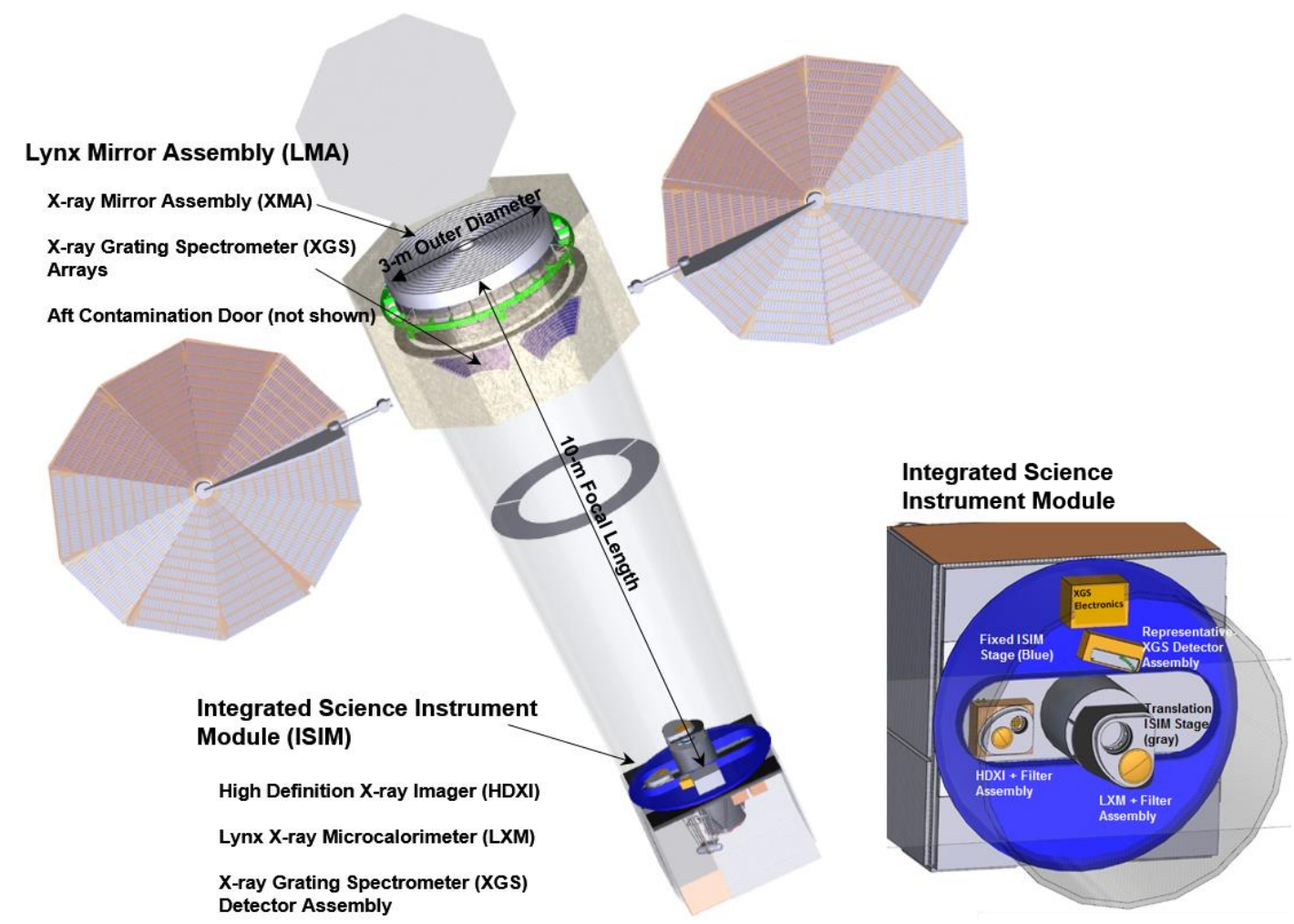

Figure 1. Lynx X-ray Telescope configuration. Lynx has a 10-m focal length and consists of a high-resolution, 3-meter diameter, large area X-ray mirror assembly with pre- and post-collimators (XMA) surrounded by the spacecraft bus and complemented by an instrument suite that includes HDXI, LXM, and XGS. 


\subsection{X-Ray Mirror Assembly}

The most stringent requirement on the XMA is set by the Lynx science goal to observe the very first Supermassive Black Holes and associating them unambiguously with the first galaxies that JWST will observe ${ }^{19}$.

On-axis angular resolution $\sim 0.5$ arcsecond (HPD) is required to avoid source confusion at the faintest fluxes and to uniquely associate X-ray sources with high-redshift optical and near-IR galaxies. A mirror effective area of $2 \mathrm{~m}^{2}$ at $1 \mathrm{keV}$ and a FOV with arcsecond or better imaging extending to at least 10 arcminutes off-axis is required to adequately sample the population of black hole seeds at high redshift well within the mission lifetime. Lynx will enable a 100-fold increase in survey depth over the deepest Chandra fields, whereas Athena will be confusion- and background-limited before even reaching the current Chandra deep field sensitivity (Figure 2).

\subsection{Science Instruments}

Just as critical as the mirror assembly, Lynx requires a detector capable of direct imaging black hole seeds in deep extragalactic

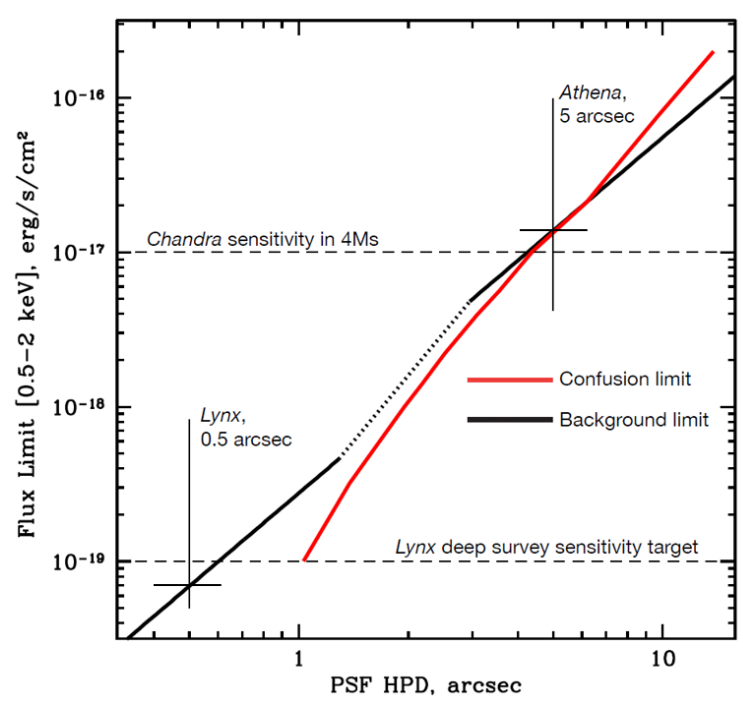

Figure 2. Sensitivity vs. angular resolution is shown, indicating that Lynx will not be confusion limited at the required sensitivity for deep surveys of the first SMBHs.

fields, taking full advantage of the mirror assembly angular resolution and FOV. In addition, this detector must have excellent soft X-ray spectral response to these (redshifted) sources and have moderate spectral resolution across the $\sim 0.2$ to $\sim 10 \mathrm{keV}$ band to deduce thermodynamic properties of the hot gas in galactic halos and other extended objects. A silicon active pixel sensor array of fine pixel scale $(0.3$ arcsecond) and large format ( 23 arcminutes x 23 arcminutes FOV), such as HDXI, is capable of meeting these requirements ${ }^{2}$.

Lynx also requires a non-dispersive imaging spectrometer that can spatially resolve Active Galactic Nucleus (AGN) feedback signatures from surrounding hot gas and jets in galaxies, groups, and clusters on 1 arcsecond or finer scales. This detector must resolve starburst-driven winds in low-redshift galaxies at high spectral resolution $(\sim 0.3 \mathrm{eV})$ over $\sim 1$ arcminute FOVs at 1 arcsecond imaging resolution; map metallicity gradients (better than $5 \mathrm{eV}$ resolution over 5 arcminute FOV) in circumgalactic, group, and galaxy cluster fields; and survey young Supernova Remnants (SNRs) in Local Group galaxies. This will be accomplished with a Lynx-unique microcalorimeter, such as LXM, which is designed to have multiple sensor arrays that meet these combinations of spectral, spatial, and FOV requirements (Figure 3$)^{5}$.

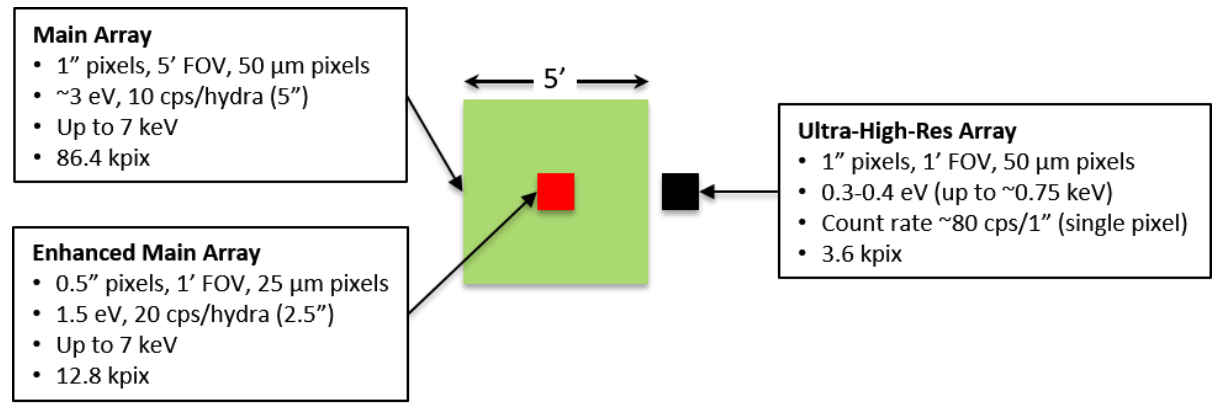

Figure 3. Baseline layout of the LXM array. There are three different regions of the array corresponding to three different pixel types, each with different properties as labeled.

Lynx must also have an instrument capable of characterizing warm gas in galactic halos out to their virial radius. This can be accomplished by absorption line studies of background AGNs requiring a high-spectral resolution $(\mathrm{R}=5,000)$ dispersive grating spectrometer such as $\mathrm{XGS}^{6}$, sensitive in the $0.2-2.0 \mathrm{keV}$ band, capable of $1 \mathrm{~m} \AA$ sensitivity in key absorption lines of OVII and OVIII. 


\subsection{Trade Studies}

The Lynx mission concept was formulated, and is being refined as a result of multiple trade studies on the telescope configuration (e.g., XMA diameter and focal length for different configurations and fixed versus extendable bench), telescope technologies (e.g., X-ray mirror technologies and detector filters), and mission design parameters (e.g., launch vehicle, orbit). Of these, one of the most critical is the trade study on the X-ray optics technology.

Multiple, actively funded, X-ray mirror technologies will be able to meet the Lynx science requirements. Deciding which of these technologies should be used for integration into the Design Reference Mission (DRM) requires careful scrutiny, as each technology has unique advantages and challenges, and each has a different development path for maturation. These technologies include Full-Shell Optics, Silicon Meta-shell Optics, and Adjustable Segmented Optics. Challenges common to all three technologies and state-of-the-art TRL are given in Table 1. Selection of a mirror technology for the DRM does not indicate which technology should be used for flight. That decision would only be made after selection of the Lynx mission, at which point each of these technologies, and perhaps others, would be assessed.

Table 1. X-ray Technologies considered for the Lynx DRM concept include Full-Shell Optics, Silicon Meta-Shell Optics, and Adjustable Segmented Optics. These three technologies are all expected to meet TRL 3 or TRL 4 by mid-2020. TRL Milestones were generated by the trade study team, and are used to evaluate, in a consistent way, the technologies relative to one another. The TRL 4 milestone is shown.

\begin{tabular}{|c|c|c|c|}
\hline Technology & Lynx Requirements & Challenges (examples) & TRL \\
\hline $\begin{array}{l}\text { High-resolution, large- } \\
\text { area, lightweight } X- \\
\text { ray grazing incidence } \\
\text { mirror assembly } \\
\quad \text { Full Shell }\end{array}$ & $\begin{array}{l}\text { - } 2 \mathrm{~m}^{2} \text { Effective Area at } 1 \mathrm{keV} \\
\text { - } 0.5 \text { arcsecond on-axis, on-orbit } \\
\text { system angular resolution } \\
\text { - Minimum grasp of } 600 \mathrm{~m}^{2} \\
\text { arcmin }{ }^{2} \text { at }<1 \text { arcsecond } \\
\text { resolution, at } 1 \mathrm{keV} \\
\text { - } 3 \mathrm{~m} \text { maximum outer diameter } \\
\text { for the XMA to maintain } \\
\text { flexibility in launch vehicle } \\
\text { selection without } \\
\text { compromising science }\end{array}$ & $\begin{array}{l}\text { - Low areal cost and } \\
\text { mass } \\
\text { - Maintain high } \\
\text { precision figure } \\
\text { through coating, post- } \\
\text { fabrication figure } \\
\text { correction, alignment, } \\
\text { and mounting } \\
\text { - Maintain performance } \\
\text { on-orbit }\end{array}$ & $\begin{array}{l}\text { - All technologies are at TRL > } 2 \\
\text { and are approaching a TRL3 to } \\
\text { TRL } 4 \\
\text { - NASA Definition TRL 4: A low } \\
\text { fidelity system or component } \\
\text { breadboard is built and operated to } \\
\text { demonstrate basic functionality } \\
\text { and critical test environments, and } \\
\text { associated performance } \\
\text { predictions are defined relative to } \\
\text { the final operating environment }\end{array}$ \\
\hline
\end{tabular}

Silicon Meta-Shell
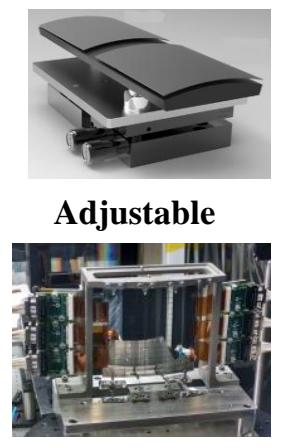

TRL 4 Milestone: Must demonstrate a credible technology development path to the required onorbit performance of the Lynx Mirror Assembly. Demonstrations must be traceable to the on-orbit performance requirement in the operational environment.

A credible demonstration of these Wolter-Schwarzschild optics must comprise the following:

- Realistic end-to-end error budget for Lynx Telescope angular resolution.

- Laboratory demonstration of measured angular resolution of mirror elements performing less than a factor of 3 away from their required performance (as stated in the error budget), executed under the following conditions:

- An X-ray test of a single coated, co-aligned p-s mirror pair or a mounted single, coated full shell using a breadboard lab mount must be demonstrated. Mirrors must have nominal thickness consistent with their point design.

- Functional breadboard mounting and all essential hardware elements (such as fixture to hold and transport full shell elements) demonstrated.

- Full shell demonstration of the alignment of a single primary shell, aligned to optical axis as defined by the mount.

- Models, Analogies, or Lab Demonstrations

- All elements related to the as-corrected mirror error contributions (e.g. coatings, thermal, g-release, etc.) must be validated.

The Lynx Team opted to use the Kepner-Tregoe ${ }^{20}$ decision-making strategy for this trade study. This strategy uses a systematic approach to reaching group consensus on key differentiating criteria to satisfy a decision statement formulated by the stakeholders. The stakeholders for this study are the members of the Lynx STDT, and the decision 
statement, or goal, is for the trade study team to recommend one DRM concept mirror architecture to focus the design for the Lynx final report and to identify all feasible alternates.

Trade study criteria includes Science, Technical, and Programmatic requirements. Each of these is broken down into two categories: absolute 'Musts' and relative 'Wants'. The 'Musts', of which there are 8 criteria (Table 2), are required to be met and are pass/fail. The 'Wants', of which there are 18 criteria (not shown here), have relative weightings and offer a comparative assessment between the technologies. Risks and opportunities are identified during this process and are an integral part of the evaluation.

Table 2. Lynx mirror technology trade criteria Kepner-Tregoe 'Musts'. The 18 'Wants' are not shown.

\begin{tabular}{|l|l|}
\hline Science & Optical performance meets the requirements flowing down from Science Traceability Matrix \\
\hline \multirow{4}{*}{ Technical } & Credible roadmap from today's status to predict flight on-orbit performance \\
\cline { 2 - 2 } & Performance modeling tools related to current results are demonstrated to be credible \\
\cline { 2 - 2 } & Repeatable fabrication process based on current status \\
\cline { 2 - 3 } & Credible error budget that flows down to each mirror element \\
\cline { 2 - 3 } & Expected to survive launch \\
\hline \multirow{3}{*}{ Programmatic } & Credible plan to meet TRL 4-6 \\
\cline { 2 - 3 } & Produce the mirror assembly within the Program schedule allocation \\
\hline
\end{tabular}

Each of the Kepner-Tregoe criteria is evaluated by a large team of experts chartered by the Lynx STDT. The recommended option, upon review by STDT and acceptance by the STDT Chairs, will serve as the reference design for the Lynx mission concept. All other feasible architectures identified in the trade process will be included in the Lynx final report. The evaluation team is a mixture of individuals both external and internal to the Lynx Program. They are volunteers from industry, the Lynx Science and Technology Definition Team, Universities, and other NASA Centers. Many of these individuals were heavily involved in the formulation and construction of Chandra, and have or are currently working on large flight Programs. The process is facilitated by G. Blackwood at NASA JPL, who has no affiliation with the technologies under consideration. A recommendation to the Lynx STDT is planned for early August of 2018 .

\section{LYNX MISSION}

\subsection{A Journey to SE-L2}

Based on a preliminary Program schedule, Lynx is planning to launch in the mid-2030s and the current assumption (still under evaluation) is that Lynx will be integrated onto a Heavy class (expendable or recoverable) vehicle that will launch from NASA Kennedy Space Center. Following a transfer trajectory insertion (TTI) maneuver, Lynx will be inserted into the 800,000-km semi-major axis halo orbit around the SE-L2 libration point and operate for 5 years with consumables for 20 years. The launch to orbit timeline and delta-v budget is shown in Figure 4.

Several orbits were analyzed for Lynx, including SE-L2, Drift-away, Lunar Distant Retrograde Orbit (LDRO), Chandratype Orbit (CTO), and Transiting Exoplanet Survey Satellite (TESS)-like. After careful consideration, SE-L2 was selected because it provides: 1) essentially no eclipsing, 2) a stable thermal environment, 3) avoidance of trapped radiation belts, 4) fewer maneuvers for orbit insertion and thus, relatively smaller propulsion system, and 5) a high observing efficiency of better than $85 \%$. The observing efficiency is the percentage of actual time Lynx will spend on science observations and takes into account-estimated times for slewing, thermal and vibrational stabilization, and calibration. 


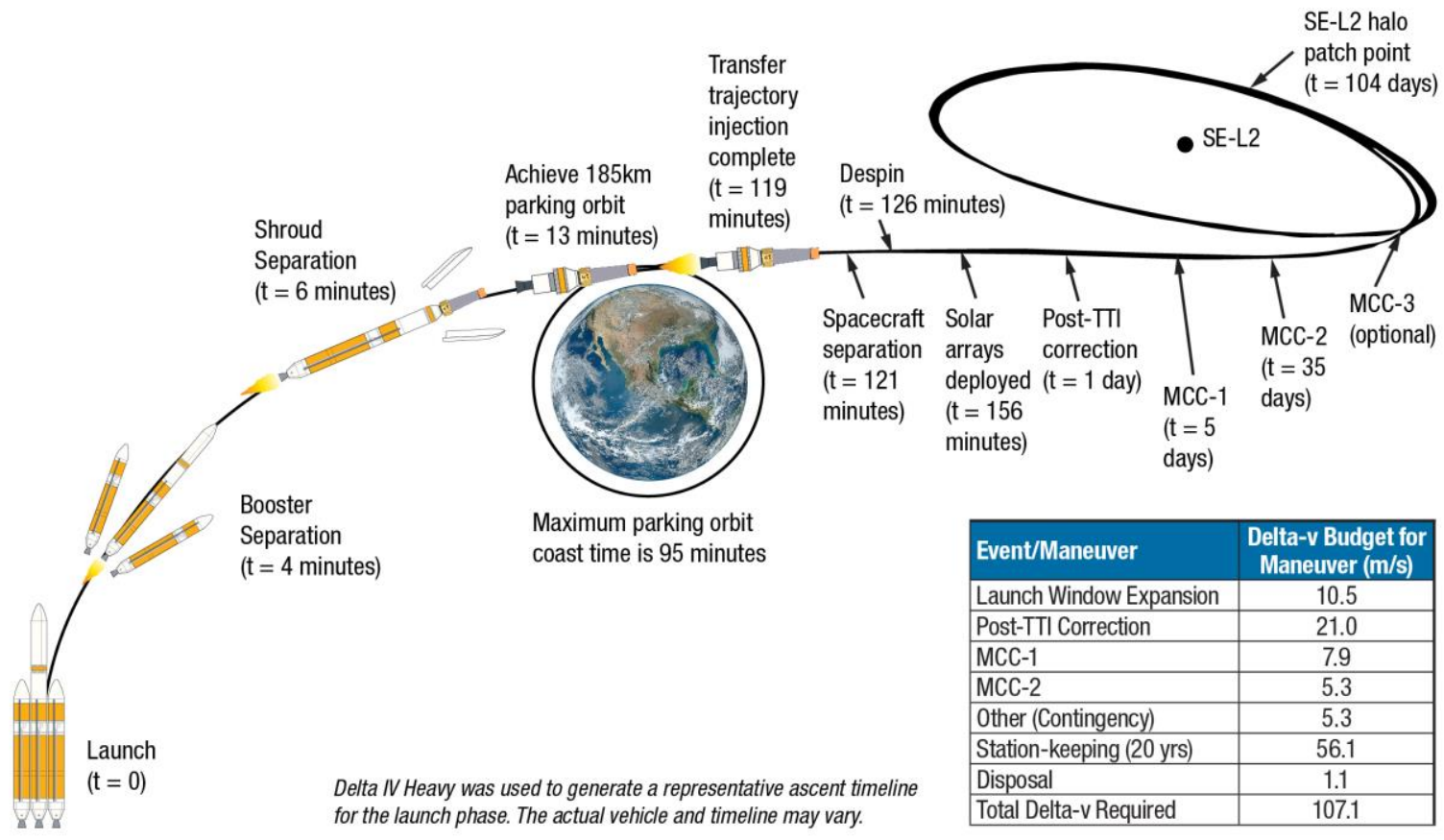

Figure 4. Launch to orbit timeline and delta-v budget.

\subsection{Spacecraft Design and Operations}

This timeline assumes launch on a Delta IV Heavy vehicle. Even though the Delta IV Heavy is not expected to be available in the 2030s, it is assumed to be representative of expected capability (and not necessarily cost) of the generic Heavy class vehicles in the 2030s. The Lynx concept team is also working with the SLS Program at MSFC to determine configuration options for launching Lynx on the SLS vehicle as a co-manifested payload. This option would provide considerable launch vehicle cost savings for the Lynx mission as launch vehicle costs are limited to integration services only. The current Lynx configuration easily meets the 10,000-kg and 7.2-m-diameter co-manifested payload envelope, but not the 8.4-m length limitation. To enable this option, the Lynx team is performing a trade study to determine the cost and risk associated with utilizing an extendable optical bench such that the observatory will fit within the SLS comanifested payload envelope (as well as within an Intermediate-class launch vehicle envelope, mass allowing).

\subsection{Science Operations}

Following on-orbit activation and checkout, Lynx will operate primarily in Normal Mode, conducting an autonomous pre-planned science program. A typical scientific observing timeline includes a series of maneuvers between targets, target acquisition, and data collection. In this mode, the focal plane science instruments are either in a data collection or standby configuration, and the observatory attitude is maintained by the PCAD system under control of the onboard computer. Normal spacecraft operations such as switching focal plane instruments, instrument calibrations, momentum unloading, ground contacts, and recorder data playback all take place in Normal Mode.

All Normal Mode operations are preplanned using a scheduling process that seeks to maximize the time on-target while accommodating all necessary spacecraft operations. The mission schedule plan will be used to generate spacecraft and instrument commands, which are then uplinked to the spacecraft and stored. A sufficient number of commands will be loaded to assure autonomous operation for 72 hours. Stored command loads can be interrupted and updated as needed to accommodate Target of Opportunity ( $\mathrm{ToO}$ ) requests (and emergencies). It is anticipated that $\mathrm{ToO}$ requests may require up to 24 hours to initiate and review new command sequences, depending on spacecraft (thermal, power, momentum, and pointing) constraints, minimization of maneuver error, and the frequency of ground contact.

On-orbit calibration observations are performed as part of the Normal Mode science operations. A set of standard celestial targets will be determined for calibration use. These targets will be periodically observed to monitor LMA, 
science instrument, and aspect system performance. In addition, HDXI and LXM contain in-situ calibration sources that are mounted on their filter assemblies. These will allow calibration data acquisition when those instruments are not carrying out celestial observations. As with other X-ray observatories such as Chandra, time allocations for calibration is expected to be about $5 \%$ of available observing time early in the mission and to decrease to 2-3\% subsequently.

\section{INTEGRATED APPROACH}

An integrated analysis of the Lynx architecture has been initiated via an industry CAN partnership that involves NGAS, Ball Aerospace, and Harris Corp. participation. This study enables refinements to the current design, by considering the integrated observatory system, and producing an error budget for the on-orbit payload performance. Integrated studies include assessing the alignment of the Lynx XMA to the focal plane, alignment of the grating arrays to the focal plane, potential thermal and mechanical instabilities on the optical bench and impact on interface design, thermal gradients on the XMA, aspect system design and accommodation, and exported disturbances based on dynamic models on-orbit. This error budget will be used to update the observatory design, until all payload performance requirements are met. During this process, trades will be identified to optimize performance, cost, and schedule for the fully integrated system ${ }^{16}$.

\section{SUMMARY}

The Lynx architecture was chosen to meet a demanding, yet realizable, science case to observe the first supermassive black holes in the Universe, trace the state of matter in cosmic structures, and to characterize the formation and evolution of stars and their local environments, including their planetary systems. The Lynx design is streamlined, employing relatively mature technologies for a concept phase and baselines standard spacecraft elements and heavy-class launch vehicles. The approach to design is integrative and system oriented, and focused on achieving the required on-orbit performance. Further, this approach will apply the appropriate lessons learned from previous missions to lower risk. The Lynx concept (artist conceptual drawing Figure 5) will be capable of flagship science for a cost that is compatible with a balanced Astrophysics portfolio.

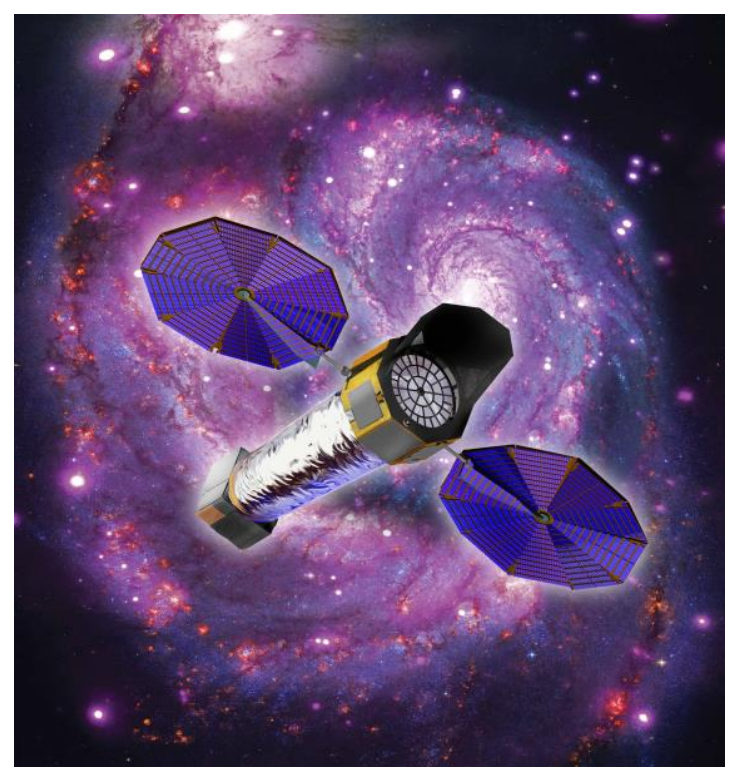

Figure 5. Conceptual design for Lynx based on the current designed architecture [Credit: David O. Miller]. 


\section{ACKNOWLEDGEMENTS}

The authors would like to acknowledge the hard work of the entire Lynx Team, including: the Science and Technology Definition Team; science, technology (optics, calibration, and instrument teams), and communications working group members; Advanced Concept Office team at MSFC; Instrument Design Lab team at GSFC; the Exploration Program Office at JPL; Lynx Mirror Assembly Trade study members; Lynx Study Office members and engineering support at MSFC and the SAO; and all others who have supported and are continuing to support Lynx. We would also like to thank all of our CAN partners, including Creare LLC, Lockheed-Martin, Hypres, Inc., and Luxel for their contributions. Most of the Lynx team members participate through contributed time and/or cost. This dedication makes Lynx possible.

\section{REFERENCES}

[1] Kouveliotou, C., Agol, E., Batalha, N., Bean, J., Bentz, M., Cornish, N., Dressler, A., Figueroa-Feliciano, E., Gaudi, S., Guyon, O., Hartmann, D., Kalirai, J., Niemack, M., Ozel, F., Reynolds, C., Roberge, A., Sheth, K., Straughn, A., Weinberg, D., Zmuidzinas, J., "Enduring quests-daring visions (NASA astrophysics in the next three decades)." arXiv preprint arXiv:1401.3741 (2014).

[2] Falcone, A. D., Kraft, R. P., Bautz, M. W., Gaskin, J. A., Mulqueen, J. A., Swartz, D. A., "The high definition X-ray imager (HDXI) instrument on the Lynx x-ray surveyor," Proc. SPIE AT\&I (2018), In Press.

[3] Bautz, M. W., Foster, R. F., LaMarr, B. J., Malonis, A., Progozhin, G. Y., Miller, E. D., Grant, C. E., Burke, B., Cooper, M., Leitz, C., Schuette, D., Suntharalingam, V., "Toward fast, low-noise, low-power digital CCDs for Lynx and other high-energy astrophysics missions," Proc. SPIE AT\&I (2018), In Press.

[4] Kenter, A., Kraft, R., Gauron, T., Amato, S., "Advancing the technology of monolithic CMOS detectors for use as x-ray imaging spectrometers," Proc. SPIE 10397 (2017).

[5] Bandler, S. R., DiPirro, M. J., Eckart, M. E., Sakai, K., Smith, S. J., Yoon, W., Bennett, D. A., Mates, J., Swetz, D. S., Ullom, J. N., Irwin, K. D., McCammon, D., Figueroa-Feliciano, E., Ryu, K. K., Zeiger, B., Olson, J., "The Design of the Lynx x-ray microcalorimeter (LXM),” Proc. SPIE AT\&I (2018), In Press.

[6] McEntaffer, R., et al., "The Lynx off-plane x-ray grating spectrograph,” Proc. SPIE AT\&I (2018), In Press.

[7] Günther, H. M., Heilmann, R., “An x-ray transmission grating spectrometer for Lynx,” Proc. SPIE AT\&I (2018), In Press.

[8] Civitani, M. M., Basso, S., Ghigo, M., Holyszko, J., Pareschi, G., Vecchi, G., Parodi, G., Kiranmayee, K., Davis, J., Elsner, R., Swartz, D. A., “Lynx optics based on full monolithic shells: design and development,” Proc. SPIE AT\&I (2018), In Press.

[9] Gubarev, M., Kolodziejczak, J. K., Griffith, C., Roche, J., Smith, W. S., Kester, T., Atkins, C., Arnold, W., Ramsey, B. D., "Development of a direct fabrication technique for full-shell x-ray optics," Proc. SPIE 9905 (2016).

[10] Zhang, W. W., et al., "Next generation astronomical x-ray optics: high resolution, light weight, and low cost," Proc. SPIE AT\&I (2018).

[11] Reid, P. B., et al., "Development of adjustable x-ray optics for the Lynx mission concept," Proc. SPIE AT\&I (2018), In Press.

[12] Arenberg, J., Matthews, G., Atkinson, C., Cohen, L., Golisano, C., Havey, K., Hefner, K., Jones, C., Kegley, J., Knollenberg, P., Lavoie, T., Oliver, J., Plucinsky, P., Tananbaum, H., Texter, S., Weisskopf, M. C., "Lessons We Learned Designing and Building the Chandra Telescope Chandra Lessons Learned," Proc. SPIE 9144 (2014).

[13] Weisskopf, M. C., "The Chandra X-ray Optics,” Optical Engineering, 51(1) (2011).

[14] http://chandra.harvard.edu/

[15] Public Law 111-267-Oct. 11, 2010, 124 Stat. 2833, Sec. 804. In-Space Servicing, https://www.congress.gov/

[16] Arenberg, J. W., Allen, L. N., Mooney, J., Purcell, W., Jordan, S., "Considerations for the development of Lynx," Proc. SPIE AT\&I (2018), In Press.

[17] Arenberg, J. W., Allen, L., N., Mooney, J., "Options for the implementation of the Lynx mirror assembly,” Proc. SPIE AT\&I (2018), In Press.

[18] Gaskin, J. A., Allured, R., Bandler, S. R., Basso, S., Bautz, M. W., Baysinger, M. F., Biskach, M. P., Boswell, T. M., Capizzo, P. D., Chan, K-W., Civitani, M., Cohen, L. M., Cotroneo, V., Davis, J. M., Deroo, C. T., DiPirro, M. J., Dominguez, A., Fabisinski, L. L., Falcone, A. D., Figueroa-Feliciano, E., Garcia, J., Gelmis, K. E., Heilmann, R. K., Hopkins, R. C., Jackson, T., Kilaru, K., Kraft, R. P., Liu, T., McClelland, R. S., McEntaffer, R. L., 
McCarley, K. S., Mulqueen, J. A., Ozel, F., Pareschi, G., Reid, P. B., Riveros, R. E., Rodriguez, M. A., Rowe, J. W., Saha, T. T., Schattenburg, M. L., Schnell, A. R., Schwartz, D. A., Solly, P. M., Suggs, R. M., Sutherlin, S. G., Swartz, D. A., Trolier-McKinstry, S., Tutt, J. H., Vikhlinin, A., Walker, J., Yoon, W., Zhang, W. W., "Lynx Mission Concept Status,” Proc. SPIE 10397 (2017).

[19] Windhorst, R. A., Cohen, S. H., Jansen, R., Conselice, C., Yan, H., "How JWST can measure first light, reionization and galaxy assembly," New Astronomy Reviews, 50 (1-3), 113-120 (2006).

[20] http://www.kepner-tregoe.com/ 